\title{
AJUSTE DE MODELO REOLÓGICO PARA ESMALTE CERÂMICO FORMULADO COM FRITA CERÂMICA DE DIFERENTE GRANULOMETRIA
}

\author{
A. L. M. F. SILVA*, A. M. P. F. FELIPE e J. A. S. SOUZA \\ Universidade Federal do Pará, Departamento de Engenharia Química \\ andremileo@ufpa.br ${ }^{*}$
}

Artigo submetido em maio/2016 e aceito em setembro/2016

DOI: $10.15628 /$ holos.2016.4596

\section{RESUMO}

Esmaltes cerâmicos são camadas finas e contínuas de constituição fundamentalmente vítrea, formadas a partir de uma mistura de matérias-primas a qual se funde sobre a superfície de uma matriz. As fritas cerâmicas aparecem como matéria-prima majoritária desse tipo de revestimentos, os quais exibem a natureza física e química de vidros, insolúveis e impermeáveis a líquidos e gases. A produção de esmaltes com qualidade e especificações técnicas para o uso como revestimentos cerâmicos requer estudo de propriedades relevantes inerentes aos processos de formulação e aplicação dos esmaltes cerâmicos, de forma a se alcançar efeitos e características desejados no produto final. $O$ estudo visou o entendimento da influência da granulometria e composição química da frita cerâmica transparente no comportamento reológico das suspensões de esmalte cerâmico, parâmetro importante para assegurar a qualidade do processo de produção e aplicação dos esmaltes cerâmicos. A formulação de esmalte cerâmico com frita de menor granulometria apresentou um comportamento reológico mais viscoso com maiores tensões de escoamento em relação a formulação de maior granulometria. O modelo Hershel Bulkley foi o que melhor se ajustou ao comportamento reológico dos esmaltes cerâmicos em relação aos modelos de Bingham e Ostwald de Waele.

PALAVRAS-CHAVE: Frita transparente, Esmalte Ceramico, Viscosidade, Reologia.

\section{RHEOLOGICAL MODEL FIT FOR CERAMIC GLAZE MADE WITH CERAMIC FRIT OF DIFFERENT GRAIN SIZE}

\begin{abstract}
Ceramic Glazes are thin and continuous layers of glass constitution basically formed from a mixture of raw materials which is melted on the surface of a matrix. The ceramic chips appear to majority feedstock of this type coatings which exhibit chemical and physical nature of glass, insoluble and impermeable to liquids and gases. The production of enamels and quality specifications for use as ceramic tile requires the study of material properties inherent in the formulation and application of ceramic glaze, so as to achieve desired effects and characteristics in the final product. The study aimed at
\end{abstract}

understanding the influence of particle size and chemical composition of the ceramic frit transparent on the rheological behavior of ceramic glaze suspensions, important parameter to ensure the quality of the production process and application of ceramic glazes. The ceramic enamel frit formulation with smaller grain size had a more viscous rheological behavior with higher yield stresses over the formulation of larger granulometry. Herschel Bulkley model was the best fit to the rheological behavior of ceramic glazes in respect of models of Bingham and Ostwald de Waele.

KEYWORDS: Transparent fries, ceramic glaze, Viscosity, Rheology. 


\section{INTRODUÇÃO}

As fritas cerâmicas são materiais vítreos com composição química adequada à necessidade de utilização como matérias-primas de revestimentos cerâmicos, esses materiais são fabricados por indústrias especializadas a partir da fusão da mistura de diferentes matérias-primas. As fritas são os componentes mais importantes da maioria dos esmaltes industriais. Após a queima esses revestimentos conferem o aspecto vítreo à matriz sobre a qual são aplicados. Além disso, a aplicação de esmalte tem por finalidade aprimorar a estética, tornar a peça impermeável a líquidos e gases, aumentar a resistência mecânica e melhorar ou proporcionar outras características (DURAN,2002).

A correta aplicação do esmalte sobre o corpo cerâmico e consequentemente a qualidade da cobertura vítrea formada possuem forte dependência das propriedades reológicas da suspensão de esmalte. Em vista disso o conhecimento e a adequação do comportamento reológico dos esmaltes cerâmicos se tornam fundamentais para a obtenção de vidrados com qualidade elevada (MELCHIADES,2009).

Devido a influência de parâmetros físicos como o tamanho e a orientação das partículas, além da complexidade da composição química das fritas, haja vista o elevado número de óxidos que geralmente estão presente em suas formulações, a completa previsão das características e do comportamento dos revestimentos formulados a partir destas matérias-primas se torna um desafio para as indústrias do setor cerâmico. Assim, aspectos relevantes às etapas de elaboração e aplicação desses materiais, com diversidade e qualidade compatíveis com a evolução do setor cerâmico, tem sido objeto de estudo (HOTZA,1998).

$\mathrm{Na}$ prática industrial, as fritas ainda hoje são desenvolvidas mediante procedimentos empíricos. Sendo assim a não adequação dos processos físicos e químicos atualmente utilizados na preparação para o uso industrial dessa matéria-prima podem tornar o processo produtivo oneroso. Em virtude disso, estudos sobre a influência das características físicas e das composições químicas das fritas no comportamento e qualidade dos esmaltes produzidos, tornaram-se uma atividade de fundamental importância para o setor principalmente na etapa de formulação e aplicação dos esmaltes cerâmicos (SERPA,2007).

A busca pela redução dos custos na produção de esmaltes, sem prejuízo às propriedades técnicas adequadas ao seu emprego se reflete no desenvolvimento de novas formulações e técnicas para obtenção e utilização das fritas cerâmicas (SILVA,2016)

O entendimento do comportamento reológico das suspensões de esmalte é importante principalmente em virtude das exigências dos métodos de aplicação a serem utilizados. As características físicas, o incremento, a redução ou até mesmo ausência total de determinados óxidos na composição da frita cerâmica, podem ocasionar alterações significativas em propriedades reológicas, como a viscosidade dos esmaltes obtidos a partir desta matéria-prima. 0 que por sua vez poderá ocasionar resultados indesejados nas etapas subseqüentes do processo produtivo. 


\section{MATERIAIS MÉTODOS}

\subsection{Caracterização química das matérias-primas}

A frita cerâmica transparente, Borax e a Argila foram submetidas a caracterização química através da técnica de fluorescência de raios-X utilizando espectrômetro WDS sequencial, modelo Axios Minerals PANalytical, com tubo de raios-X cerâmico e anodo de ródio.

\subsection{Moagem e classificação granulométrica da frita cerâmica transparente}

A frita transparente passou por um processo de moagem, nos tempos de 5, 10, 30, 60, 90 e 120 minutos, em condições operacionais especificadas na Tabela 1.

A moagem tem o objetivo reduzir as partículas do material, aumentando sua área específica e conseqüentemente, a interação entre as matérias-primas utilizadas (GLENN,1980).

A cominuição da frita transparente e demais matérias-primas foi realizada a seco utilizando um moinho de bolas de escala piloto com dimensões de 260 × 350 mm (diâmetro x comprimento), o moinho de bolas foi carregado com $1 \mathrm{~kg}$ de frita transparente para aproximadamente $3 \mathrm{~kg}$ de bolas, e operou a uma velocidade de rotação igual a $87 \%$ de sua velocidade crítica para cada ensaio. Amostras da frita transparente foi submetida a análise granulométrica com o objetivo de determinar o tamanho em função do tempo de moagem. As condições operacionais de moagem segue a metodologia SAMPAIO (2007).

Tabela 1: Condições de moagem estabelecidas para a etapa de cominuição.

\begin{tabular}{c|c|c|c}
\hline \multicolumn{3}{c}{ CARACTERÍSTICAS DA CARGA DE BOLAS UTILIZADAS NO TESTE } \\
\hline No DE BOLAS & $\begin{array}{c}\text { DIÂMETRO } \\
(\mathbf{c m})\end{array}$ & PESO $(\mathbf{g})$ & VOLUME $\left(\mathbf{c m}^{\mathbf{3}}\right)$ \\
\hline $\mathbf{8}$ & 3.676 & 1825.84 & 208 \\
$\mathbf{1 2}$ & 2.879 & 1021.2 & 150 \\
$\mathbf{9}$ & 1.563 & 211.5 & 18 \\
\hline \multicolumn{2}{c}{ Total } & 3058.54 & 376 \\
\hline
\end{tabular}

\subsection{Formulação dos Esmaltes Cerâmicos}

Após a moagem e classificação granulométrica da frita transparente, na classificação particular passantes nas peneiras de 106 e $63 \mu \mathrm{m}$ para a formulação dos esmaltes cerâmicos A e $B$, respectivamente.

Tabela 2: Composição principal do esmalte cerâmico formulado.

\begin{tabular}{c|c|c|c}
\hline COMPONENTE & QUANTIDADE & FRAÇÃO SÓLIDA \% & FRAÇÃO TOTAL \% \\
\hline Frita & $40 \mathrm{~g}$ & 79,4 & 50,89 \\
Borax & $9,6 \mathrm{~g}$ & 19,0 & 12,21 \\
Argila ( $\left.\mathrm{D}_{\mathrm{i}}<100 \mathrm{Mesh}\right)$ & $0,8 \mathrm{~g}$ & 1,6 & 1,02 \\
Carboximetilcelulose $(\mathrm{CMC})$ & $0,7 \mathrm{ml}$ & - & 0,89 \\
Solução água - cola 10:1 & $0,4 \mathrm{ml}$ & - & 0,51 \\
Água & $27,10 \mathrm{ml}$ & - & 34,48 \\
\hline
\end{tabular}


A composição dos esmaltes formulados no trabalho foi adaptada segundo NORTON (1973). A Tabela 2 apresenta a composição mássica do esmalte cerâmico variando apenas a granulometria da frita nas formulações $A$ e B. As matérias-primas devidamente pesadas foram misturadas em um gral durante 10 minutos, em seguida foram transferidas para um Béquer onde foram adicionas a água e as soluções de CMC e Água-cola.

\subsection{Ensaios reológicos}

Os estudos reológicos das suspensões de esmaltes A e B, com teor massico de sólidos em $65 \%$ foram realizados a temperatura ambiente de 25 ㅇ C, em viscosímetro modelo Haake VT 550 com sensor tipo cilindro coaxiais SV1. O viscosímetro modelo Haake VT 550 apresenta respostas com baixo desvio para sistemas particulados com teor mássico acima de 55 \% (SILVA, 2015).

\section{RESULTADOS E DISCUSSÃO}

\subsection{Composição química das matérias-primas}

A Tabela 3 apresenta a composição química da frita transparente, do bórax e da argila, materiais usados na formulação dos esmaltes cerâmicos.

Tabela 3: Composição química da frita transparente, bórax e da argila.

\begin{tabular}{c|c|c|c}
\hline ELEMENTO & FRITA(\%) & BORAX(\%) & ARGILA(\%) \\
\hline $\mathrm{SiO}_{2}$ & 74,48 & 0,23 & 64,18 \\
$\mathrm{P}_{2} \mathrm{O}_{5}$ & 0,06 & ------- & ------- \\
$\mathrm{Al}_{2} \mathrm{O}_{3}$ & 6,95 & 6,27 & 16,56 \\
$\mathrm{Fe}_{2} \mathrm{O}_{3}$ & 0,13 & ------- & 6,38 \\
$\mathrm{MgO}$ & 0,43 & ------ & 0,74 \\
$\mathrm{BaO}$ & 2,02 & ------ & ------- \\
$\mathrm{CaO}$ & 2,82 & ------ & 0,23 \\
$\mathrm{ZnO}$ & 0,73 & ------ & -------- \\
$\mathrm{PbO}$ & 0,2 & ------ & ----- \\
$\mathrm{K} 2 \mathrm{O}$ & 2,06 & ------ & 1,79 \\
$\mathrm{TiO}_{2}$ & ------- & 1,02 \\
$\mathrm{Na}_{2} \mathrm{O}$ & 9,78 & ---- & 0,6 \\
$\mathrm{PF}$ & 0,35 & 23,92 & 8,5 \\
\hline
\end{tabular}

Pela Tabela 3 observa-se que a frita transparente, apresenta composição química adequada a formulação de esmaltes cerâmicos. O resultado indica um elevado percentual de $\mathrm{SiO}_{2}(74,48 \%)$, o qual, durante o processo de produção das fritas, é introduzido sob a forma de quartzo, areia quartzosa, areia feldspática, feldspato e caulim. O resultado da fluorescência mostrou ainda um teor considerável de $\mathrm{Al}_{2} \mathrm{O}_{3}$ (6,95\%). Outras composições importantes observadas na frita transparente foram as dos óxidos modificadores de rede como $\mathrm{CaO}, \mathrm{K}_{2} \mathrm{O}$ e $\mathrm{ZnO}$. As relações entre os teores desses óxidos podem ser determinantes para o entendimento da viscosidade obtidas nos esmaltes cerâmicas.

A argila revelou a presença de $\mathrm{SiO}_{2}, \mathrm{Al}_{2} \mathrm{O}_{3}$ e $\mathrm{Fe}_{2} \mathrm{O}_{3}$, principais responsáveis pela adequação do coeficiente de retração do revestimento ao coeficiente da peça sobre a qual o mesmo será aplicado. A análise do Borax utilizado revelou a presença em grande quantidade de $\mathrm{Na}_{2} \mathrm{O}(69,58 \%)$ o qual tem a função de agir como fundente durante a etapa de queima o que caracteriza esse 
componente como capaz de adequar o intervalo de temperatura de fusibilidade do revestimento em relação as necessidades operacionais.

\subsection{Análise granulométrica}

A Figura 1 mostra a distribuição granulométrica da frita cerâmica transparente nos diversos tempos empregados na etapa de moagem.

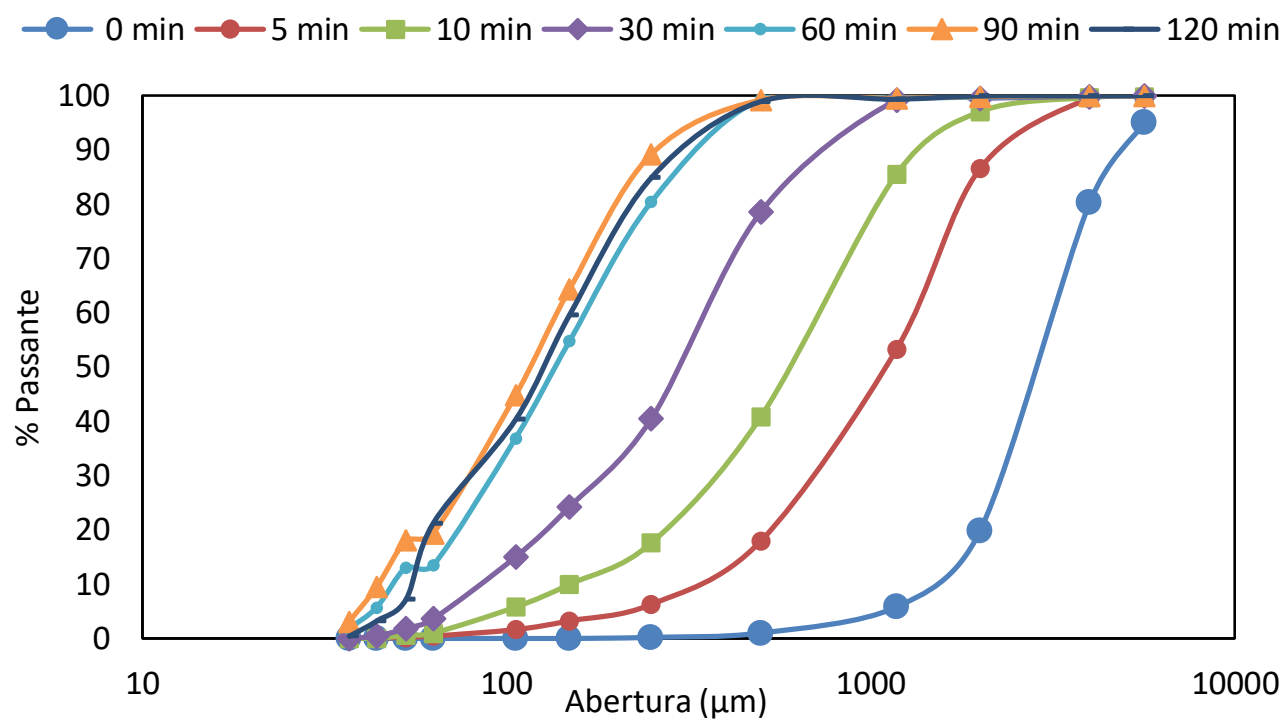

Figura 1: Distribuição granulométrica da frita cerâmica transparente de acordo com os tempos de moagem.

Pela Figura 1 observa-se que com o passar do tempo de moagem em minutos está havendo a diminuição da granulometria da frita cerâmica o que confirma que as condições operacionais de moagem está correta.

Para evidenciar a distribuição granulométrica da frita cerâmica transparente de acordo com os tempos de moagem a Figura 2 apresenta o diâmetro médio $\left(D_{50}\right)$ em $\mu \mathrm{m}$ da frita em função do tempo de moagem.

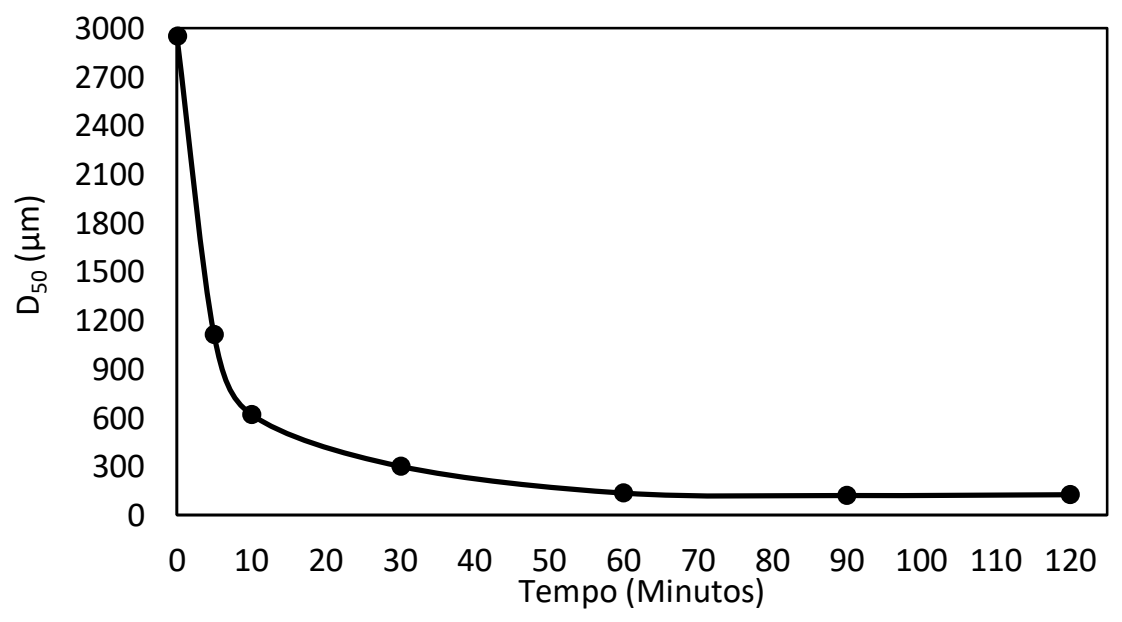

Figura 2: $d_{50}$ função do tempo de moagem. 
Pela Figura 2 os dados indicam a eficiência da operação até o tempo de 60 minutos, a partir do qual, observa-se uma tendência à estabilização nos valores dos tamanhos médios de partícula em função do tempo em minutos da etapa de cominuição.

Para as condições estabelecidas apos 60 minutos a variação nos tamanhos médios das partículas de frita não sofre mais redução significativa em seu diâmetro a ponto de justificar o gasto de energia com a operação de cominuição. Portanto, logo para as condições operacionais estabelecidas e característica físicas iniciais da matéria-prima, a continuação da etapa de moagem, para tempos superiores a 60 minutos, geraria um maior gasto energético ao processo de produção sem diminuição apreciável no $\mathrm{d}_{50}$ do material.

Essa queda na eficiência de moagem provavelmente é ocasionada por diversos fatores, dentre eles destacam-se: as restrições operacionais da operação moagem tendo em vista o tamanho e forma inicial das partículas, dureza e tendência a aglomeração do material.

\subsection{Curva de moagem}

Visando aperfeiçoar a obtenção de frita transparente na quantidade e especificações físicas necessárias a formulação dos esmaltes, utilizaram-se os dados gerados na análise granulométrica para construir uma curva de eficiência da frita transparente em moinho de bolas.

A Figura 3 é a curva de moagem da frita transparente nas condições estabelecidas para o teste. E foi obtida traçando-se o gráfico \% Passantes versus tempo de moagem.

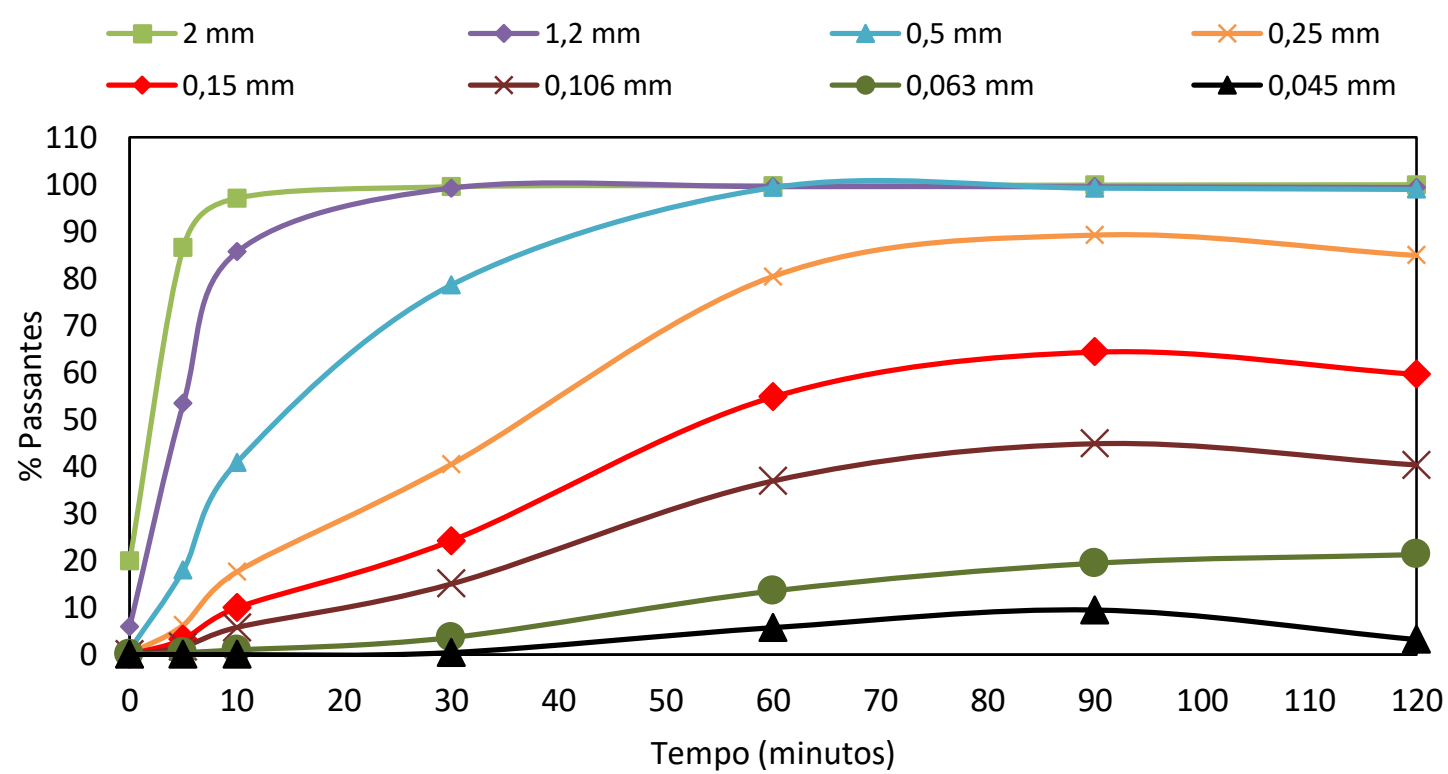

Figura 3: Curva de moagem da frita cerâmica transparente em moinho de bola.

A partir da Figura 3 foi possível determinar de maneira eficiente o tempo de moagem necessário para a obtenção da maior quantidade passante possível da frita transparente em determinada peneira com as respectivas aberturas em $\mathrm{mm}$.

A Tabela 4 mostra os passantes nas peneiras para as formulações A e B. 
Tabela 4: Passantes para as formulações A e B

\begin{tabular}{c|c}
\hline ESMALTE & TAMANHO DE PARTÍCULA DA FRITA TRANSPARENTE UTILIZADA \\
\hline A & $D_{\mathrm{i}}<106 \mu \mathrm{m}$ \\
B & $D_{\mathrm{i}}<63 \mu \mathrm{m}$ \\
\hline
\end{tabular}

Observa-se pela Tabela 4 que o esmalte A possui granulometria maior que o esmalte B. $\mathrm{O}$ esmalte A possui frita cerâmica em sua composição passante em peneira de 100 mesh com abertura de $106 \mu \mathrm{m}$ já o esmalte $B$ possui frita passante em peneira de 250 mesh de abertura de $63 \mu \mathrm{m}$

\subsection{Comportamento Reologico dos esmaltes cerâmicos}

O estudo do comportamento reológico dos esmaltes cerâmicos $A$ e $B$, permitiu a verificação da adequação entre o comportamento experimental das suspensões e os principais modelos reológicos conhecidos.

A Tabela 5 apresenta os valores dos parâmetros reológicos bem como das correlações obtidas para os esmaltes $A$ e $B$, ajustados aos modelos reológicos da literatura.

Tabela 5: Valores dos parâmetros reológicos e correlação para os esmaltes A e B segundo os principais modelos reológicos factíveis.

\begin{tabular}{l|c|c|c}
\hline MODELO REOLÓGICO & EQUAÇÃO & ESMALTE A & ESMALTE B \\
\hline \multirow{2}{*}{ Herschel - Bulkley } & \multirow{2}{*}{$\tau=\tau_{0}+K \dot{\gamma}^{n}$} & $\mathrm{R}^{2}=0,9988$ & $\mathrm{R}^{2}=0,9980$ \\
& & $\tau_{0}=0,3320$ & $\tau_{0}=0,5491$ \\
& & $\mathrm{~K}=2,082$ & $\mathrm{~K}=2,467$ \\
& & $\mathrm{n}=0,4194$ & $\mathrm{n}=0,3855$ \\
\hline Bingham & $\tau=\tau_{0}+\eta_{a 0} \dot{\gamma}$ & $\mathrm{R}^{2}=0,9595$ & $\mathrm{R}^{2}=0,9547$ \\
& & $\tau_{0}=6,660$ & $\tau_{0}=7,988$ \\
& & $\eta_{\mathrm{ap}}=0,07130$ & $\eta_{\mathrm{ap}}=0,08438$ \\
\hline \multirow{2}{*}{ Ostwald de Waele } & $\mathrm{R}=0,9980$ & $\mathrm{R}^{2}=0,9952$ \\
& \multirow{2}{*}{$\tau=k \dot{\gamma}^{n}$} & $\mathrm{~K}=2,248$ & $\mathrm{~K}=2,733$ \\
& \multicolumn{2}{c}{$\mathrm{n}=0,4079$} & $\mathrm{n}=0,4178$ \\
\hline
\end{tabular}

De acordo com a Tabela 5 é possível observar que os modelos reológicos de HerschelBulkley e de Ostwald de Waele, apresentaram valores muito próximos. Contudo o modelo de Ostwald de Waele acaba não sendo representativo por omitir a tensão limite de escoamento. 0 modelo de Hershel-Bulkley engloba fatores que o modelo de Ostwald de Waele não revela, por isso torna-se o mais representativo para descrever o comportamento dos esmaltes A e B. A suspensão de esmalte $B$ apresentou uma tensão limite de escoamento $\left(\tau_{0}=0,5491\right)$, superior ao apresentado pelo esmalte $A$. Isso é explicado fato de que a frita transparente utilizada na formulação do esmalte $B(\mathrm{Di}<63 \mu \mathrm{m})$, apresenta uma maior superfície específica do que aquela utilizada na formulação do esmalte $\mathrm{A}(\mathrm{Di}<106 \mu \mathrm{m})$, o que diminui a sua capacidade de escoamento.

\subsection{Curvas de fluxo dos esmaltes A e B}

A Figura 4 apresenta as curvas de fluxo obtidas para os esmaltes A e B. 


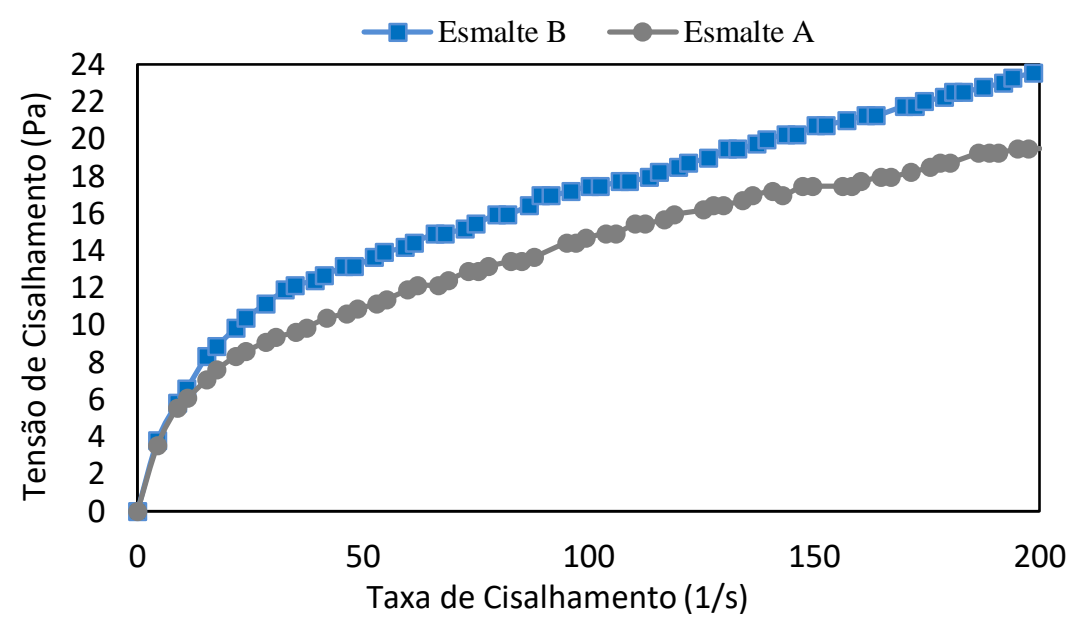

Figura 4: Curvas fluxo dos esmaltes A e B.

As curvas da Figura 4 mostram o comportamento reológico característicos das suspensões A e B, segundo o modelo reológico de Herschel-Bulkley. Segundo BRAGANÇA (2008) esse comportamento é explicado pela existência de uma estrutura interna, ocasionada pela formação de uma rede de ligação entre as partículas através de forças polares e ou de Van der Waals. Além disso, a presença da Carboximetilcelulose em dissolução modifica as características das suspensões, promovendo ainda mais o comportamento plástico causando também um aumento na viscosidade dos esmaltes.

3.6 Curvas de viscosidade dos esmaltes A e B

A Figura 5 mostra as curvas de viscosidade obtidas para os esmaltes A e B.

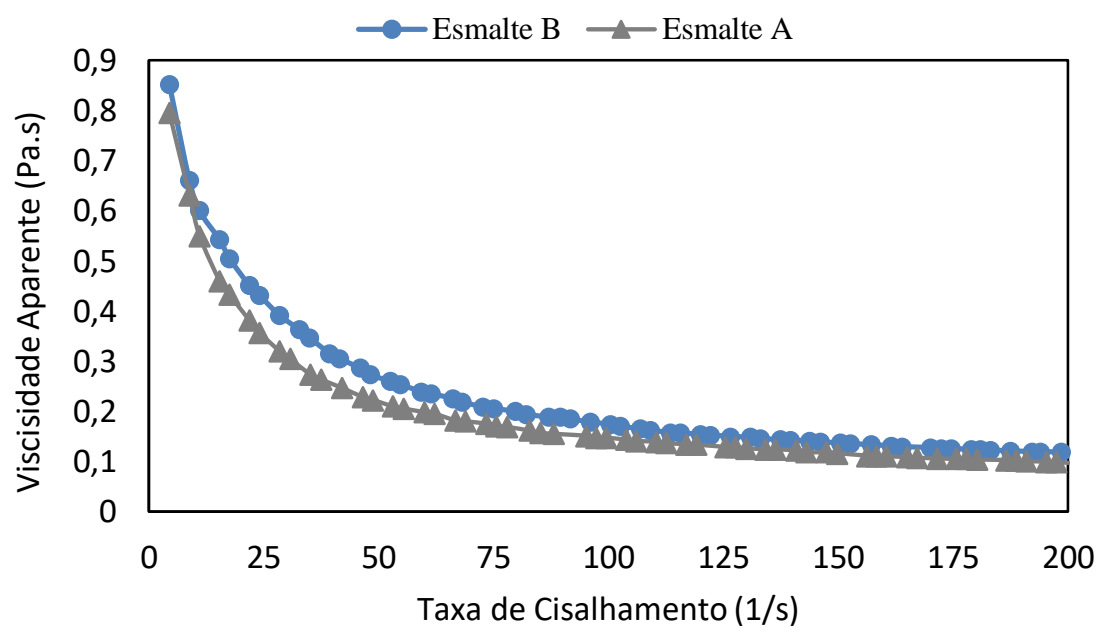

Figura 5: Curvas de viscosidade para os esmaltes A e B.

As curvas da Figura 5 mostram ainda que, mantendo fixa a concentração de sólidos e o valor da taxa de cisalhamento, o esmalte $B$ é mais viscoso quando comparada ao esmalte $A$, pois apresenta maior quantidade de finos, aumentando assim, sua viscosidade. Este fato pode ser explicado pela maior área superficial das partículas finas, que favorece as interações de van der Waals, inclusive com maior probabilidade de ocorrer a formação de aglomerados, nos quais as partículas primárias estão unidas e aprisionam água em seu interior. Como esta água não está 
disponível para o fluxo, a suspensão se comporta como se a fração volumétrica de sólidos fosse maior, o que aumenta a viscosidade (WANG,2006).

A distribuição de tamanhos de partícula do esmalte exerce grande influência sobre a viscosidade, a fixação ao substrato, a velocidade de deposição e a espessura do recobrimento.

\section{CONCLUSÃO}

Conclui-se que na formulação de esmaltes cerâmicos quanto menor a granulometria da frita cerâmica maior será a viscosidade e a tensão cisalhante para escoar o esmalte. O esmalte B por possui menor granulometria de partículas em relação ao esmalte $A$ apresentou comportamento reológico mais viscoso o que leva ao consumo de mais energia em um processo de escoamento para aplicação de esmalte em peças cerâmicas além de aumenta a espessura de deposito do esmalte em peças. Tanto o esmalte $A$ quanto o esmalte $B$ possuem comportamento reológico regido pelo modelo de Hershel Bulkley. Todos os dois esmaltes possuem comportamento reológico ajustável o que garante boa modelagem nos processos de escoamento na industria cerâmica.

\section{AGRADECIMENTOS}

Agradecimento a CAPES, ao programa de pós-graduação em Engenharia Química da Universidade Federal do Pará por criarem boas condições ao desenvolvimento de pesquisa.

\section{REFERÊNCIAS}

1. BRAGANÇA, A.C.C. Avaliação de reagentes alternativos para a substituição da cal em sistemas de longa distância de polpa de minério de ferro. 2008. 116f. Dissertação (Mestrado em Engenharia Metalúrgica e de Minas) - Universidade Federal de Minas Gerais. Belo Horizonte, 2008.

2. DURAN, A., HEVIA, R., CENTRITTO, N., OLIVEIRA, A. P. N., BERNARDIN, A. M. Introducción a los esmaltes cerâmicos. Madrid: FaenzaEditrice Ibérica, 2002. 224 p.

3. GLENN, I. STELZER, e KLUG, E.D. Handbook of watersoluble gums and resins. New York: McGraw Hill, 1980: p. 4-2 a 4-28.

4. WANG, HE, M., Y. \& FORSSBERG, E. Parameter Studies on the Rheology of Limestone Slurries. International Journal of Mineral Processing. 78, 2006, p.63-77.

5. HOTZA, D. et al. Influência da adição de defloculante, ligante e partículas de alumina no comportamento reológico de suspensões a base de frita e caulim. Química Nova, 21(4), 1998.

6. MELCHIADES, F.G. et al. Formulação de Fritas Cerâmicas com Auxílio da Técnica de Planejamento Estatístico de Experimentos. Cerâmica Industrial, v.14, n. 3, Maio/Junho, 2009.

7. NORTON, F. H. Introdução a tecnologia cerâmica. São Paulo, Editora Edgard Blucher, 1973.

8. SAMPAIO, J. A.; FRANÇA, S.C.A.; BRAGA, P.F.A. Tratamentos de minérios: Práticas Laboratoriais. /Ed. - Rio de Janeiro: CETEM/MCT, 2007.

9. SERPA, E.C.N. Obtenção de fritas cerâmicas a partir de resíduo refratário proveniente do setor 
metalúrgico. Dissertação (Mestrado em Engenharia Química) - Universidade Federal de Santa Catarina. Florianópolis, 2007.

10. SILVA, A. L. M. F.; FELIPE, A. M. P. F. ; SOUZA, J. A. S. . Estado da Arte do Emprego da Lama Vermelha como Materias-Prima Fundente na Formulação de Esmaltes Cerâmicos. Revista SODEBRAS, v. 11, p. 168-171, 2016.

11. SILVA, A. L. M. F.; CARDOSO, D. N. P. ; FELIPE, A. M. P. F. ; SOUZA, J. A. S. . Influência de aditivo PVA no comportamento reológico de argamassa elaborada a partir de resíduos Industriais.. Cerâmica Industrial (Impresso), v. 20, p. 24-29, 2015. 